\title{
BMJ Open Use of music to enhance sleep and psychological outcomes in critically ill patients: a protocol for a systematic review and meta-analysis
}

\author{
Lixia Chen (D) , ${ }^{1}$ Fang Wang, ${ }^{2}$ Jianhua Li, ${ }^{1}$ Li Cui, ${ }^{1}$ Xiaoli Liu, ${ }^{3}$ Cuihua Han, ${ }^{1}$ \\ Siqi Qu, Liang Wang, ${ }^{5}$ Daihong $\mathrm{Ji}^{1}$
}

To cite: Chen L, Wang F, Li J, et al. Use of music to enhance sleep and psychological outcomes in critically ill patients: a protocol for a systematic review and meta-analysis. BMJ Open 2021;11:e037561. doi:10.1136/ bmjopen-2020-037561

- Prepublication history for this paper is available online. To view these files, please visit the journal online (http://dx.doi. org/10.1136/bmjopen-2020037561).

LC and FW contributed equally.

LC and FW are joint first authors.

Received 07 February 2020 Revised 18 February 2021 Accepted 23 February 2021

Check for updates

(C) Author(s) (or their employer(s)) 2021. Re-use permitted under CC BY-NC. No commercial re-use. See rights and permissions. Published by BMJ.

For numbered affiliations see end of article.

Correspondence to

Daihong Ji;

yourfriend.123@163.com

\section{ABSTRACT}

Introduction Music listening is used as a nonpharmacological intervention in various populations with positive results; however, evidence for its effect on sleep and psychological outcomes in critically ill patients remains unclear. It is essential to understand the impact of music listening for critically ill patients to optimise care and minimise the risk for harm. We will assess whether music listening improves sleep and psychological outcomes in critically ill patients.

Methods and analysis We will systematically search scientific databases for relevant studies, including PubMed, Embase, CINAHL, PsycINFO, Web of Science, Scopus, ProQuest, the Cochrane Central Register of Controlled Trials, China Biological Medicine Database, China National Knowledge Infrastructure Library, Wan fang databases, VIP Database for Chinese Technical Periodicals and the Chinese Clinical Trial Registry. Databases will be searched for articles published from inception to 10 June 2020. Music therapy journals and reference lists in some articles will be handsearched. Grey literature will also be searched. We will include randomised and quasi-randomised controlled trials that used music listening to improve sleep and psychological outcomes in critically ill patients. The primary outcomes will be sleep-related outcomes, and secondary outcomes will be anxiety and depression scores and physiological outcomes. Two reviewers will independently verify study eligibility and methodological quality; disagreements will be resolved by a third reviewer or through discussion. The risk of bias will be independently determined using the Cochrane Risk of Bias Tool. The Consolidated Standards of Reporting Trials checklist will be used to examine the quality of included papers. Data will be extracted from eligible studies by two researchers. RevMan V.5.3 will be used for meta-analysis.

Ethics and dissemination This work will review existing trial data and will not introduce new patient data or interventions; therefore, ethics committee approval is not required. We will disseminate this protocol in a related peer-reviewed journal.

PROSPERO registration number CRD42019147202.

\section{INTRODUCTION}

\section{Rationale}

Sleep disturbance is a frequent problem among patients in the intensive care unit (ICU). ${ }^{2}$ Sleep quality and quantity are
Strengths and limitations of this study

- We plan to employ robust international goldstandard methodology and a comprehensive search strategy to reduce bias.

- We will assess the quality of included articles using a validated tool.

- Subgroup analyses will be performed when possible to elaborate intervention or participant characteristics correlated with increased effectiveness.

- High heterogeneity across studies may increase the difficulty in interpreting a meta-analysis.

- A limitation will be that the systematic review protocol will only include articles published in English and Chinese.

negatively affected by the ICU environment (eg, noise and lights), ${ }^{3}$ patient-care activities, symptoms of the patient's underlying illness and mechanical ventilation. ${ }^{45}$ In this context, sleep is characterised by prolonged sleep onset latency, short sleep duration, frequent awakenings, non-restorative sleep and decreased sleep efficiency (percentage of time in bed spent asleep). Sleep disturbance has been associated with numerous adverse consequences in critically ill patients, including impairments in immune function, memory, wound healing and inspiratory muscle endurance, along with higher rates of delirium and increased overall morbidity and mortality. ${ }^{6}$

An increase in psychological problems (eg, anxiety and depression) has also been found in critically ill patients. ${ }^{78}$ One study ${ }^{9}$ estimated that $70 \%-80 \%$ of critically ill patients experienced anxiety related to fear, sleeplessness, pain, discomfort, thirst and disease-related symptoms. That study also reported patients on assisted ventilation were especially prone to anxiety because of their need for frequent suctioning, inability to breathe independently 
or talk and general discomfort. In addition, around half of patients experienced a high level of depression during their ICU stay. ${ }^{9}$ Unmanaged anxiety and depression have been associated with harmful effects on disease recovery and overall well-being, including prolonged weaning from ventilation and recovery time,${ }^{10}$ increased work for breathing, fatigue, ${ }^{11}$ acute elevated blood pressure ${ }^{12}$ and an increased depression incidence in ICU survivors. ${ }^{13}$ Wewalka found that pre-existing depressive mood at the time of ICU admission was an independent risk factor for 28-day mortality among patients in the medical ICU. ${ }^{14}$

Sufficient evidence shows that sleep disturbance, anxiety and depression are detrimental to disease recovery and psychological well-being. Research has also reported an interplay of sleep disturbance, anxiety and depression. ${ }^{15}$ Anxiety and depression are both risk factors for sleep disturbance and disturbed sleep pattern increases emotional distress, which in turn lead to higher levels of anxiety and depression. ${ }^{9} 16$ Therefore, as key ICU staff, nurses need to provide effective interventions to address these issues.

Pharmacological and non-pharmacological interventions are used to manage sleep and psychological distress in the ICU. Pharmacological therapy is generally the firstline treatment. ${ }^{17}{ }^{18}$ However, pharmacological therapy has been associated with numerous adverse effects and complications, including memory loss, prolongation of mechanical ventilation, altered sleep stages, longer length of hospitalisation, tolerance, bradycardia, hypotension, residual daytime effects, dysmotility, weakness and delirium. ${ }^{19}{ }^{20}$ In addition, the medications used are expensive. To avoid these issues, researchers have developed alternative, non-pharmacological therapies to improve sleep, anxiety and depression among patients in the ICU, and positive results have been reported. ${ }^{21-23}$ The results of recent music intervention studies have drawn attention to the relationship between music and sleep in various patient groups. This resulted in increased use of music therapy and music listening (sometimes called music medicine ${ }^{24}$ ).

Music can be defined as the organisation of tone over the time, and it is one of the most pleasurable experiences for a human being. In the early 1800 s, Florence Nightingale $^{25}$ described the importance of music and its healing effect on patients; the music first implemented in hospitals was live music. With the development of the music discipline and science, more recorded music was used and played to patients using musical equipment such as portable stereos, wall-mounted speakers or devices such as MP3 players. ${ }^{26}$ Music as a non-pharmacological intervention has been adopted by medical staff, with these interventions involving different types of music, such as low volume, nature sounds, soothing music and Mozart piano recordings. As an intervention, music is relatively easy to implement, cost effective, safe and has no negative impacts. ${ }^{27}$ Music therapy and music listening are common forms of music application that are similar but have distinct features. Music therapy is defined as the clinical and evidence-based use of music to realise individualised clinical goals within a therapeutic relationship. It is conducted by certified music therapists, and centres on the dynamic musical interaction between the music therapist and the patient, verbal processing of the music experience, and implementation and alteration of music (tempo, volume and intensity) according to the patient's needs. ${ }^{28}{ }^{29}$ Music therapy consists of active and passive forms. The active form refers to therapy that needs patient participation in the process, whereas the passive form refers to therapy that comprises listening to music without participation. Irrespective of whether it is active or passive, music therapy is an evidence-based practice conducted by certified music therapists. During therapy, music therapists consider elements of music such as melody, rhythm, tempo and harmony. ${ }^{30}$ The use of environmental music therapy (EMT), which is type of music therapy, has increased in recent years. ${ }^{31} 32$ EMT involves trained, certified professionals using live music to address a chaotic intensive care environment and help to create a less tense atmosphere. They apply live music to meet the psychological, physical and cultural needs of caregivers, patients and staff in the hospital environment. A previous study verified the safety and effectiveness of EMT. ${ }^{33}$

Music listening is defined as passive listening to recorded music via any form of music playback device (eg, earphones or speakers) or listening to live music without interacting with a music therapist or theoretical framework. It can be provided by medical or healthcare professionals or self-administered by a patient, and patients may or may not be involved in selecting the music. ${ }^{34} 35$ Although music-based applications are used in both music listening and music therapy, it is important to distinguish the two interventions in clinical practice ${ }^{36}$ because of the varying levels of training in the fundamentals of music and its therapeutic applications. For example, music therapists have received specialised training in the aforementioned areas. ${ }^{24}$ The effectiveness of music therapy is mostly attributable to the active musical interaction between the patient and the music therapist, which is why numerous studies suggest music therapy is more effective than music listening. However, most patients in the ICU are critically ill and weak, and they may not have enough energy to interact with a music therapist. Music listening may therefore be preferred, as compared with music therapy, it may be used by more patients. Music listening has also been widely used to assuage emotional, physiological and psychological symptoms in various diseases, such as Parkinson's disease, ${ }^{37}$ Alzheimer's disease ${ }^{38}$ and cancer. ${ }^{39}$ A growing number of studies involving adults of all ages with various medical and surgical conditions have demonstrated the positive effects of music listening on anxiety, depression, stress and pain. ${ }^{40}{ }^{41}$ Repeated studies have specifically reported music listening improved sleep in critically ill patients. ${ }^{234243}$ Furthermore, music listening is inexpensive, relatively easy to implement and safe compared with pharmacological interventions ${ }^{27}$; these benefits are favourably received by patients. Therefore, 
music listening is a potentially viable alternative treatment option.

Clinical trials have provided support for the effectiveness of the application of music in the healthcare setting. Psychophysiological theory ${ }^{44}$ also provides clues to its mechanism of action. Music comprises many key elements, including rhythm, pitch, harmony and melody. These elements play a comprehensive role in the degree to which music can promote sleep in patients. ${ }^{45}$ Previous authors described that sleep improvement is mediated by the relaxing, distracting effect of 'soothing' music. ${ }^{46}$ Music with a slow tempo (eg, 60-80 beats/min) mirrors the heart rate and reduces neuroendocrine and sympathetic nervous system activity, resulting in relaxation. Furthermore, the peaceful atmosphere created by soothing music in the ICU setting is a mood enhancer, reduces anxiety and depression and lowers treatmentrelated stress. Other authors described the effect of music in modulating mood and emotions at the cortical level through stimulation of self-image and intellect. ${ }^{47}$ Clinical trials have also shown that listening to music reduced anxiety and stress responses, which can lead to greater relaxation and improved sleep. ${ }^{48} 49$

Research on the impact of music listening for patients in the ICU has evolved over the past 20 years, and several researchers have studied the effects of music listening on sleep and psychological outcomes in critically ill patients. For example, recent studies reported that music listening may improve stress, anxiety, ${ }^{850}$ depression ${ }^{51}$ and sleep ${ }^{5253}$ in ICU patients. As an intervention, music listening involves using different types of music to improve sleep and psychological symptoms (eg, low volume, nature sounds, soothing music, classical music). Music listening can be provided by specific tools (eg, MP3 format via earphones or loudspeaker). The choice of music may be determined by the researcher or by participants themselves. The duration, frequency and timing of music exposure have also varied among studies. Although clinical trials have been performed to investigate the effects of music listening on sleep and psychological outcomes, their safety and efficacy for critically ill patients have not been established. In addition, most of these studies used small sample sizes, ${ }^{434}$ making it nearly impossible to achieve statistically significant results. The impact of music listening may also differ because of different intervention designs (study design, method of intervention and type of music). A previous systematic review assessed the efficacy of music application for reducing anxiety among mechanically ventilated patients, ${ }^{35}$ but only included mechanically ventilated patients. A recent systematic review also evaluated the effectiveness of music therapy to reduce stress and anxiety in critically ill patients. ${ }^{55}$ In 2015 , another review reported that music therapy appeared to be safe to improve sleep; however, that study did not perform a meta-analysis and noted further randomised controlled trials were required to assess efficacy. ${ }^{56}$ The 2018 Pain, Agitation/sedation, Delirium, Immobility (rehabilitation/mobilization), and Sleep (disruption) (PADIS) guideline also suggests there is no high-quality evidence to prove that music could improve sleep in critically ill adults. ${ }^{1}$

Despite the large number of relevant studies, music listening has not been implemented as a therapeutic intervention in everyday critical care because information about its effectiveness has not been synthesised and disseminated universally. Therefore, we aim to assess the effectiveness of music listening in improving sleep, anxiety and depression in critically ill patients, and investigate relevant subgroups (ie, timing of intervention, type of intervention, severity of disease, mechanical ventilation status and study site).

\section{OBJECTIVES}

This systematic review and meta-analysis aims to integrate available scientific research on the use of music listening to promote sleep and reduce anxiety and depression for critically ill patients in the ICU. We will attempt to answer the following research questions.

1. What are the effects of music listening on sleep quality and quantity in critically ill patients?

2. What are the effects of music listening on anxiety, depression and physiological outcomes in critically ill patients?

\section{METHODS AND ANALYSIS}

This paper presents a quantitative systematic review protocol. We will follow the Preferred Reporting Items for Systematic Review and Meta-Analysis Protocols (PRISMA-P) guidelines to complete and report the study protocol. ${ }^{57}$ This systematic review protocol has been registered in PROSPERO.

\section{Patient and public involvement}

There is no patient or public involvement in this study.

\section{Inclusion/exclusion criteria}

Types of participants

Studies will be selected for inclusion if their participants meet specific criteria:

- Adult patients in the ICU (aged $\geq 18$ years).

- Conscious and clear (Glasgow Coma Scale score $\geq 14$ ).

- Ventilated or non-ventilated.

- Admitted to the ICU $\geq 24$ hours.

We will exclude studies that included participants with:

- Hearing damage.

- A diagnosis of or overt signs or symptoms of obstructive sleep apnea.

- A diagnosis of dementia or neurological disease.

- Severe signs or symptoms of psychological illness, such as hallucinations, delusions and behavioural disorders.

Types of intervention and comparison

We will include any study that considered sleep or psychological variables as outcomes of music listening combined 
with standard care versus standard care alone or standard care with other interventions in critically ill patients.

\section{Types of outcome measures}

At least one of the following outcomes must have been reported in the study.

\section{Primary outcomes}

Sleep outcomes

1. Sleep quality.

2. Sleep onset latency.

3. otal sleep time.

4. Number of awakenings.

5. Sleep efficiency (percent of time in bed spent asleep).

Sleep outcomes may be measured using a variety of methods. Subjective perception of sleep is measured through validated self-report tools, including the Richards-Campbell Sleep Questionnaire, ${ }^{58}$ Pittsburgh Sleep Quality Index ${ }^{59}$ and the Verran and SnyderHalpern Sleep Scale. ${ }^{60}$ Objective measurement of sleep is performed with polysomnography, actigraphy, bispectral index monitoring or electroencephalography.

\section{Secondary outcomes}

Psychological outcomes

1. Anxiety.

2. Depression.

We will include trials that measured psychological outcomes using standardised questionnaires with established reliability and validity, including Hospital-based Anxiety and Depression Scales, ${ }^{61}$ the Visual Analogue Scale for Anxiety, ${ }^{62}$ the Spielberger State-Trait Anxiety Inventory $^{63}$ and the Beck Anxiety Inventory. ${ }^{64}$

Physiological outcomes (heart rate, blood pressure and respiratory rate).

\section{Types of study designs}

We will include any interventional study, including randomised and quasi-randomised controlled trials.

\section{Data source and search strategy}

To identify eligible studies, we will search electronic databases, including PubMed, Embase, CINAHL, PsycINFO, Web of Science, Scopus, ProQuest, the Cochrane Central Register of Controlled Trials, China Biological Medicine Database, China National Knowledge Infrastructure Library, Wang Fang databases, VIP Database for Chinese Technical Periodicals and the Chinese Clinical Trial Registry. These databases will be searched from inception to 10 June 2020. In addition, we will hand-search music therapy journals and the reference lists of relevant articles, as well as grey literature.

A health sciences librarian will design the search. Box 1 shows the search strategy with both keywords and Medical Subject Heading terms that will be used to search PubMed; this strategy will be adapted as appropriate for other databases.

\section{Box 1 Search strategy for PubMed}

\section{Filter: Humans}

1. music [Mesh] $O R$ 'music therapy' [Mesh] OR music medicine $O R$ music $^{*}$ OR listen*

2. sleep [Mesh] OR sleep Disorders, Circadian Rhythm [Mesh] OR sleep* OR insomnia* OR wakeful ${ }^{\star}$ OR sleepless*

3. anxiety [Mesh] OR fear [Mesh] OR stress OR psychological OR depression [Mesh] OR depress ${ }^{\star}$ OR mood disorders [Mesh)

4. 2 OR 3

5. critical illness [Mesh] OR critical care [Mesh] OR 'intensive care units' [Mesh] OR ventilators, mechanical (Mesh) OR respiration, artificial [Mesh] OR intensive care OR ICU

6. 1 AND 4 AND 5

7. infant* $O R$ neonat* $O R$ infant, premature [Mesh] OR infant, newborn [Mesh] OR Intensive Care Units, pediatric [Mesh]

8. 6 NOT 7

\section{Selection of studies}

All articles retrieved through the search of the selected databases will be imported into EndNote, and duplicate references will be removed. Two members of the research team (SQ and $\mathrm{CH}$ ) will independently review the title/ abstract of each article to verify that each study meets the inclusion criteria. If a title or abstract is unclear, the two researchers will review the full article. Disagreements will be resolved by a third researcher (LCh) or through discussion until consensus is reached. The reasons for all exclusions will be recorded.

\section{Data collection and validation}

Two researchers (JL and $\mathrm{LCu}$ ) will independently extract data from the included studies using the Cochrane Collaboration Data Collection Form. ${ }^{65}$ In the event of questions about or missing data in the original text, the researchers will contact the authors to obtain the relevant data. The results of data extraction will be compared to exclude any differences, and any disagreement will be resolved by a third researcher (LCh) or through discussion and consensus.

From all included studies, we will collect the following data.

1. Research and publication information, including the title, journal (volume, page number) or if unpublished, the author, year of publication, country, setting, language of publication and funding sources.

2. Study design, including the type of design, inclusion criteria, exclusion criteria, randomisation method (including concealment and blinding) and loss to followup.

3. Characteristics of the participants, including total sample size, number of participants in the intervention and control groups, gender, age, diagnosis, disease severity (Acute Physiology and Chronic Health Evaluation (APACHE) II scores), comorbidities and mechanical ventilation status.

4. Intervention details, including type of music, control of music selection (by participant or researcher), the 
frequency, duration and timing of music listening and the format/devices used (eg, headphone and loudspeaker).

5. Outcomes, including the methods of assessment of sleep, anxiety and depression, pre-test and post-test means or change scores and SD.

\section{Methodological quality assessment}

The risk of bias and quality of the included studies will be evaluated using the Cochrane Collaboration Risk of Bias Tool ${ }^{65}$ which evaluates seven sources of study bias: (1) random sequence generation, (2) allocation concealment, (3) performance bias, (4) detection bias, (5) incomplete outcome data, (6) selective reporting and (7) other bias. Two researchers (LCh and XL) will independently grade each element as low risk, high risk or unclear risk. ${ }^{66}$ Inconsistencies will be resolved through discussion and consensus, or by a third researcher (DJ). An appropriate assessment will be completed for the quality assessment of quasi-randomised controlled trials.

\section{Data synthesis and analysis}

A meta-analysis will be conducted when there are sufficient studies showing homogeneity. Statistical analyses will be performed using RevMan V.5.3.5 software. Continuous data will be expressed with ORs and 95\% CIs. The level of heterogeneity of the included studies will be determined with the $\mathrm{I}^{2}$ statistic and $\mathrm{p}$ value. ${ }^{67}$ If there is statistical heterogeneity ( $p>0.1$ and $\left.\mathrm{I}^{2}<50 \%\right)$, a meta-analysis will be performed using a fixed-effects model. A random-effects model will be used to analyse clinical heterogeneity if $\mathrm{I}^{2}>50 \%$. Subgroup analyses will be performed by timing of intervention, type of intervention, severity of disease (APACHE II score <25, 25-35, >35), mechanical ventilation status (ventilated patients vs non-ventilated patients) and study site (surgical ICU patients vs medical ICU patients). Sensitivity analyses will be used to determine the stability of the results, and Egger's regression test and funnel plots will be used to assess potential publication bias. If data pooling is not possible, quantitative data will be presented in a narrative review using thematic summaries and tables.

\section{Validity and reliability/rigour}

The study protocol will use systematic review and metaanalytic methods and follow the Cochrane Collaboration recommendations for performing a systematic review. The results will be reported according to the PRISMA-P guidelines. ${ }^{68}$ In addition, the Consolidated Standards of Reporting Trials checklist will be used to examine the quality of the included papers.

\section{DISCUSSION}

This paper presents the protocol for a systematic review of the literature examining the effects of music listening on sleep and psychological outcomes among critically ill patients. The study will be undertaken to answer questions about the effectiveness of music listening in this population.

Properly powered, intervention studies provide strong evidence; therefore, this meta-analysis of existing evidence will permit conclusions about the efficacy of music listening on sleep, anxiety and depression among critically ill patients. Results from this study will provide recommendations for the use of music listening in this population and support nurses and other health practitioners in their promotion of mental health. In addition, by identifying existing lacunae in the literature, our results will prompt further research.

\section{ETHICS AND DISSEMINATION}

This work will review existing trial data and will not introduce new patient data or interventions; therefore, ethical committee approval is not required. This systematic review protocol will follow the PRISMA checklist. We will disseminate this protocol in a related peer-reviewed journal or at conferences.

\section{Author affiliations}

${ }^{1}$ Nursing Department, Affiliated Zhongshan Hospital of Dalian University, Dalian, China

${ }^{2}$ Hemopurification Center, The Second Affiliated Hospital of the University of South China, Hengyang, China

${ }^{3}$ Operating room, Peking University People's Hospital, Beijing, China

${ }^{4}$ School of Nursing, Dalian University, Dalian, China

${ }^{5}$ Intensive Care Unit, Sichuan Provincial People's Hospital, Chengdu, China

Acknowledgements We thank Eleanor Scharf, MSc(A), from Liwen Bianji, Edanz Editing China (www.liwenbianji.cn/ac), for editing the English text of a draft of this manuscript.

Contributors LCh, FW and LW were responsible for drafting the article or revising it critically for important intellectual content. JL and LCu conducted final approval of the version to be submitted. DJ, $\mathrm{SQ}$ and $\mathrm{CH}$ were responsible for the conception and design of the study, XL supervised the work. All authors approved the final version of the article.

Funding This work was funded by the Journal of Chinese Medical Association (CMAPH-NRI2019024) and Affiliated Zhongshan Hospital of Dalian University.

Competing interests None declared.

Patient and public involvement Patients and/or the public were not involved in the design, or conduct, or reporting, or dissemination plans of this research.

Patient consent for publication Not required.

Provenance and peer review Not commissioned; externally peer reviewed.

Open access This is an open access article distributed in accordance with the Creative Commons Attribution Non Commercial (CC BY-NC 4.0) license, which permits others to distribute, remix, adapt, build upon this work non-commercially, and license their derivative works on different terms, provided the original work is properly cited, appropriate credit is given, any changes made indicated, and the use is non-commercial. See: http://creativecommons.org/licenses/by-nc/4.0/.

ORCID iD

Lixia Chen http://orcid.org/0000-0001-9877-532X

\section{REFERENCES}

1 Devlin JW, Skrobik Y, Gélinas C, et al. Clinical practice guidelines for the prevention and management of pain, Agitation/Sedation, delirium, immobility, and sleep disruption in adult patients in the ICU. Crit Care Med 2018;46:e825-73. 
2 Hweidi IM, Nizamli FM. Stressors in intensive care units in Syria: patients' perceptions. J Res Nur 2015;20:114-26.

3 Pisani MA, Friese RS, Gehlbach BK, et al. Sleep in the intensive care unit. Am J Respir Crit Care Med 2015;191:731-8.

4 Pulak LM, Jensen L. Sleep in the intensive care unit: a review. J Intensive Care Med 2016;31:14-23.

5 Elliott R, McKinley S, Cistulli P, et al. Characterisation of sleep in intensive care using 24-hour polysomnography: an observational study. Crit Care 2013;17:R46.

6 Delaney LJ, Van Haren F, Lopez V. Sleeping on a problem: the impact of sleep disturbance on intensive care patients - a clinical review. Ann Intensive Care 2015;5:3.

7 Oh J, Sohn J-H, Shin CS, et al. Mutual relationship between anxiety and pain in the intensive care unit and its effect on medications. $J$ Crit Care 2015;30:1043-8.

8 Lee $\mathrm{C}-\mathrm{H}$, Lee $\mathrm{C}-\mathrm{Y}$, Hsu M-Y, et al. Effects of music intervention on state anxiety and physiological indices in patients undergoing mechanical ventilation in the intensive care unit. Biol Res Nurs 2017:19:137-44.

9 Treggiari-Venzi M, Borgeat A, Fuchs-Buder T, et al. Overnight sedation with midazolam or propofol in the ICU: effects on sleep quality, anxiety and depression. Intensive Care Med 1996;22:1186-90.

10 Wong HL, Lopez-Nahas V, Molassiotis A. Effects of music therapy on anxiety in ventilator-dependent patients. Heart Lung 2001;30:376-87.

11 Hetland B, Lindquist R, Chlan LL. The influence of music during mechanical ventilation and weaning from mechanical ventilation: a review. Heart Lung 2015;44:416-25.

12 Loomba RS, Arora R, Shah PH, et al. Effects of music on systolic blood pressure, diastolic blood pressure, and heart rate: a metaanalysis. Indian Heart J 2012;64:309-13.

13 Davydow DS, Gifford JM, Desai SV, et al. Depression in general intensive care unit survivors: a systematic review. Intensive Care Med 2009;35:796-809.

14 Wewalka M, Warszawska J, Strunz V, et al. Depression as an independent risk factor for mortality in critically ill patients. Psychosom Med 2015;77:106-13.

15 Ding Q, Redeker NS, Pisani MA, et al. Factors influencing patients sleep in the intensive care unit: perceptions of patients and clinical staff. Am J Crit Care 2017;26:278-86.

16 Kamdar BB, Needham DM, Collop NA. Sleep deprivation in critical illness: its role in physical and psychological recovery. $J$ Intensive Care Med 2012;27:97.

17 Feren S, Schweitzer PK, Walsh JK. Pharmacotherapy for insomnia. Handb Clin Neurol 2008;24:93-105.

18 Mofredj A, Alaya S, Tassaioust K, et al. Music therapy, a review of the potential therapeutic benefits for the critically ill. $J$ Crit Care 2016;35:195-9.

19 DeMartinis NA, Kamath J, Winokur A. New approaches for the treatment of sleep disorders. Adv Pharmacol 2009;57:187-235

20 Arroliga AC, Thompson BT, Ancukiewicz M, et al. Use of sedatives, opioids, and neuromuscular blocking agents in patients with acute lung injury and acute respiratory distress syndrome. Crit Care Med 2008;36:1083

21 Kozasa EH, Hachul H, Monson C, et al. Mind-Body interventions for the treatment of insomnia: a review. Rev. Bras. Psiquiatr. 2010;32:437-43.

22 Han L, Li JP, Sit JWH, et al. Effects of music intervention on physiological stress response and anxiety level of mechanically ventilated patients in China: a randomised controlled trial. $J$ Clin Nurs 2010;19:978-87.

23 RF H, Jiang XY, Chen J. Non-pharmacological interventions for sleep promotion in the intensive care unit. Cochrane Db Syst Rev 2015;10.

24 Yinger OS, Gooding L. Music therapy and music medicine for children and adolescents. Child Adolesc Psychiatr Clin N Am 2014;23:535-53.

25 Bulechek GM, McCloskey JC. Nursing interventions: treatments for nursing diagnoses. Am J Nurs 1985;85:1350.

26 Lee W-P, Wu P-Y, Lee M-Y, et al. Music listening alleviates anxiety and physiological responses in patients receiving spinal anesthesia. Complement Ther Med 2017;31:8-13.

27 Cepeda MS, Carr DB, Lau J. Music for pain relief. Cochrane Db Syst Rev 2004;2.

28 Golino AJ, Leone R, Gollenberg A, et al. Impact of an active music therapy intervention on intensive care patients. Am J Crit Care 2019;28:48-55.

29 Mofredj A, Alaya S, Tassaioust K, et al. Music therapy, a review of the potential therapeutic benefits for the critically ill. J Crit Care 2016;35:195-9.
30 Bernatzky G, Presch M, Anderson M, et al. Emotional foundations of music as a non-pharmacological pain management tool in modern medicine. Neurosci Biobehav Rev 2011;35:1989-99.

31 Canga B, Hahm CL, Lucido D, et al. Environmental music therapy: a pilot study on the effects of music therapy in a chemotherapy infusion suite. Music Med 2012;4:221-30.

32 Zhang JW, Doherty MA, Mahoney JF. Environmental music in a hospital setting: considerations of music therapists and performing musicians. Music Med 2018;10:71-9.

33 Chang-Lit W, Loewy J, Fox J. Project sleep: the role and effect of a comprehensive, multidisciplinary music therapy quality improvement program. J Sleep Dis Res 2018;1:26-41.

34 Chan MF, Wong ZY, Thayala NV. The effectiveness of music listening in reducing depressive symptoms in adults: a systematic review. Complement Ther Med 2011;19:332-48.

35 Bradt J, Dileo C. Cochrane review: music interventions for mechanically ventilated patients. J Evid Based Med 2015;8:56.

36 Hansen IP, Langhorn L, Dreyer P. Effects of music during daytime rest in the intensive care unit. Nurs Crit Care 2018;23:207-13.

37 Cochen De Cock V, Dotov DG, Ihalainen P, et al. Rhythmic abilities and musical training in Parkinson's disease: do they help? NPJ Parkinsons Dis 2018;4:8.

38 Guess H. Alzheimer's disease and the impact of music therapy: a systematic literature review. JMURJ 2018;5:2.

39 Bradt J, Dileo C, Magill L, et al. Music interventions for improving psychological and physical outcomes in cancer patients. Cochrane Database Syst Rev 2016;8:CD006911.

40 Marie C, Wendy C, Mary Anne H. Music and its effect on anxiety in short waiting periods: a critical appraisal. J Clin Nurs 2010;14:145-55.

41 Chan MF, Chan EA, Mok E. Effects of music on depression and sleep quality in elderly people: a randomised controlled trial. Complement Ther Med 2010;18:150-9.

42 Ryu M-J, Park JS, Park H. Effect of sleep-inducing music on sleep in persons with percutaneous transluminal coronary angiography in the cardiac care unit. J Clin Nurs 2012;21:728-35.

43 CP S, Lai HL, Chang ET. A randomized controlled trial of the effects of listening to non-commercial music on quality of nocturnal sleep and relaxation indices in patients in medical intensive care unit. $J$ Adv Nurs 2013;69:1377-89.

44 Chang H-K, Peng T-C, Wang J-H, et al. Psychophysiological responses to sedative music in patients awaiting cardiac catheterization examination: a randomized controlled trial. $J$ Cardiovasc Nurs 2011;26:E11-18

45 Loewy J. Music therapy as a potential intervention for sleep improvement. Nat Sci Sleep 2020;12:1.

46 Lai H-L, Li Y-M, Hui-Ling L. The effect of music on biochemical markers and self-perceived stress among first-line nurses: a randomized controlled crossover trial. J Adv Nurs 2011;67:2414-24.

47 Koelsch S, Jäncke L. Music and the heart. Eur Heart $J$ 2015;36:3043-9.

48 Zhang J-M, Wang P, Yao J-xin, et al. Music interventions for psychological and physical outcomes in cancer: a systematic review and meta-analysis. Support Care Cancer 2012;20:3043-53.

49 Dileo C, Bradt J. Music therapy: applications to stress management. principles and practice of stress management. 3rd edn. New York: Guilford, 2007.

50 Cooke M, Chaboyer W, Schluter P, et al. The effect of music on discomfort experienced by intensive care unit patients during turning: a randomized cross-over study. Int $J$ Nurs Pract 2010;16:125-31.

51 Yanhong Z, Meng J. Clinical research of the effect of music therapy on ICU patients with loneliness, anxiety and depression. China Health Industry 2013.

52 Su C-P, Lai H-L, Chang E-T, et al. A randomized controlled trial of the effects of listening to non-commercial music on quality of nocturnal sleep and relaxation indices in patients in medical intensive care unit. $J$ Adv Nurs 2013;69:1377-89.

53 RF H, Jiang XY, Hegadoren KM. Effects of earplugs and eye masks combined with relaxing music on sleep, melatonin and cortisol levels in ICU patients: a randomized controlled trial. Crit Care 2015;19:1-9.

54 Papathanassoglou EDE, Hadjibalassi M, Miltiadous P, et al. Effects of an integrative nursing intervention on pain in critically ill patients: a pilot clinical trial. Am J Crit Care 2018:27:172-85.

55 Umbrello M, Sorrenti T, Mistraletti G, et al. Music therapy reduces stress and anxiety in critically ill patients: a systematic review of randomized clinical trials. Minerva Anestesiol 2019;85:886.

56 Sandoval CP. Nonpharmacological interventions for sleep promotion in the intensive care unit. Crit Care Nurse 2017:37:100-2.

57 Shamseer L, Moher D, Clarke M, et al. Preferred reporting items for systematic review and meta-analysis protocols (PRISMA-P) 2015 elaboration and explanation. BMJ2015;349:97647. 
58 Richards KC, O'Sullivan PS, Phillips RL. Measurement of sleep in critically ill patients. J Nurs Meas 2000;8:131-44.

59 Buysse DJ, Reynolds CF, Monk TH, et al. The Pittsburgh sleep quality index: a new instrument for psychiatric practice and research Psychiatry Res 1989;28:193-213.

60 Snyder-Halpern R, Verran JA. Instrumentation to describe subjective sleep characteristics in healthy subjects. Res Nurs Health 1987;10:155-63.

61 Zigmond AS, Snaith RP. The hospital anxiety and depression scale. Acta Psychiatr Scand 1983;67:361.

62 Hornblow AR, Kidson MA. The visual analogue scale for anxiety: a validation study. Aust N Z J Psychiatry 1976;10:339-41.

63 Marteau TM, Bekker H. The development of a six-item short-form of the state scale of the Spielberger State-Trait anxiety inventory (STAI). Brit J Clin Psychol 1992;31:301-6.
64 Fydrich T, Dowdall D, Chambless DL. Reliability and validity of the Beck anxiety inventory. J Anxiety Disord 1992;6:55-61.

65 Higgins J, Green S. Cochrane Handbook for systematic reviews of interventions. Cochrane Db Syst Rev 2011;2011.

66 Higgins JPT, Altman DG, Gøtzsche PC, et al. The Cochrane collaboration's tool for assessing risk of bias in randomised trials. BMJ 2011;343:d5928

67 Higgins JPT, Thompson SG. Quantifying heterogeneity in a metaanalysis. Stat Med 2002;21:1539-58.

68 Knobloch K, Yoon U, Vogt PM. Preferred reporting items for systematic reviews and meta-analyses (PRISMA) statement and publication bias. J Craniomaxillofac Surg 2011;39:91-2. 